\title{
Lutte Contre Le Tabagisme Et Les Maladies Non Transmissibles En Côte d'Ivoire
}

\author{
Aloko-N'guessan Kouadio Joël-Henri Gilles \\ Doctorant en communication pour le développement, \\ Université Félix-Houphouët Boigny, Côte d’Ivoire
}

Doi:10.19044/esj.2018.v14n17p235 URL:http://dx.doi.org/10.19044/esj.2018.v14n17p235

\begin{abstract}
WHO is in charge of the fight against smoking and its consequences in the world. This institution makes recommendations to its member states in order to help them contain the scourge of smoking. The present study therefore evaluates the level of integration of these various recommendations of the WHO in the context of the fight against smoking in Côte d'Ivoire. It starts from the observation that the fight against smoking in Ivory Coast has produced for several years mixed results. From a methodological point of view, the approach adopted in this study is essentially qualitative. It consisted of conducting semi-structured interviews, a quantitative thematic content analysis and a bibliographic synthesis. Through this approach, we have achieved results that highlight the inadequacy of the legal framework and tobacco control tools in Côte d'Ivoire and the weakness of financial resources allocated to the fight against smoking in this country.
\end{abstract}

Keywords: Fight, smoking, non-communicable diseases, Côte d'Ivoire

\section{Résumé}

La lutte contre le tabagisme et ses conséquences dans le monde est l'une des prérogatives de l'Organisation Mondiale de la Santé (OMS). Cette institution édicte dans ce sens des recommandations à l'intention de ses Etats membres afin de leur permettre de contenir le fléau que constitue le tabagisme. La présente étude évalue donc le niveau d'intégration de ces différentes recommandations de l'OMS dans le cadre de la lutte contre le tabagisme en Côte d'Ivoire. Elle part du constat que la lutte contre le tabagisme en Côte d'Ivoire produit depuis plusieurs années des résultats mitigés. Sur le plan méthodologique, la démarche adoptée dans la présente étude est essentiellement qualitative. Elle a consisté à réaliser des entretiens semi-directifs, une analyse de contenu thématique quantitative et une synthèse bibliographique. Par le biais de cette démarche, nous avons obtenu 
des résultats qui mettent en lumière une insuffisance du cadre juridique et des instruments de lutte contre le tabagisme en Côte d'Ivoire et une faiblesse des ressources financières allouées à la lutte contre le tabagisme dans ce pays.

Mots-clés : Lutte, Tabagisme, maladies non transmissibles, Côte d'Ivoire

\section{Introduction}

Le tabagisme représente la première cause de décès évitables dans le monde selon l'Organisation Mondiale de la Santé (OMS, 2016) qui comptabilise 6 millions de décès causés annuellement par le tabagisme dans le monde (OMS, 2016). En outre, l'institution estime les pertes financières mondiales liées à la consommation de produits tabagiques à 1400 milliards de dollars (OMS, 2017).

Le tabagisme est en nette progression en Afrique. Ainsi, certaines estimations font état d'une hausse de la prévalence du tabagisme en Afrique qui passera de $16 \%$ à $22 \%$ entre 2010 et 2030 sans l'adoption de mesures appropriées (Network of African Science Academies [NASAC], 2014). Cette évolution de la prévalence tabagique en Afrique laisse entrevoir un péril sanitaire sur ce continent à travers une augmentation importante des cas de maladies non transmissibles dont le tabagisme représente l'une des causes les plus importantes.

En Côte d'Ivoire également, certaines études font état d'une forte prévalence du tabagisme. Dans ce sens, le Consortium pour la Recherche Economique et Sociale (CRES, 2013) estime que 5000 personnes meurent chaque année des conséquences du tabagisme dans le pays dont la prévalence tabagique est estimée à environ $15 \%$ selon cette institution (CRES, 2013).

Afin de lutter contre les conséquences du tabagisme, la Côte d'Ivoire a intensifié son action dans le domaine de la lutte antitabac à travers la création du Programme National de Lutte contre le Tabagisme, l'Alcoolisme et les autres Addictions (PNLTA) en 2008, la ratification de la conventioncadre de l'OMS pour la lutte antitabac en 2010 ou encore l'adoption en 2012 d'un décret d'interdiction de fumer dans les lieux publics (CRES, 2013). Il existe par ailleurs une plateforme de la société civile, le Réseau des ONG Actives pour le Contrôle du tabac en Côte d'Ivoire (ROCTA-CI ${ }^{40}$, qui mène des actions en faveur de la lutte antitabac en collaboration avec le PNLTA (CRES, 2013). Cependant, ces différentes actions entreprises dans le cadre de la lutte contre le tabagisme n'ont pas réussi à changer les habitudes de

${ }^{40}$ Le ROCTA-CI est le principal réseau d'ONG active dans la lutte contre le tabagisme en Côte d'Ivoire. 
consommation de tabac en Côte d'Ivoire et le tabagisme est par ailleurs en hausse dans le pays (CRES, 2013). Dans ce sens, il importe de déterminer les facteurs qui empêchent une baisse de la prévalence du tabagisme en Côte d'Ivoire en dépit des actions mises en œuvre dans le pays. Pour ce faire, il convient de s'attarder sur la forme que prend en Côte d'Ivoire, l'application des recommandations de l'OMS en matière de lutte antitabac quand on sait que de nombreux pays, y compris certains pays africains, sont parvenus à réduire la prévalence du tabagisme en mettant en œuvre de manière rigoureuse, lesdites recommandations (Centre de Recherches pour le Développement International [CRDI], 2011; Arvers, 2017) ${ }^{41}$.

\section{Méthode de l'étude}

La présente étude prend en compte les actions menées dans le cadre de la lutte antitabac entre 2003 et 2016 en Côte d'Ivoire. Ce choix se justifie par le fait que la lutte contre le tabagisme dans le monde s'est considérablement accentuée avec l'adoption de la convention cadre de l'OMS pour la lutte antitabac au cours de l'année 2003 (Must et Efroymson, 2003). Nos différentes enquêtes ont été menées entre juillet 2016 et janvier 2018.

Dans le cadre de la collecte de nos données, nous avons eu recours aux méthodes qualitatives de l'entretien semi-directif, de la recherche documentaire et de l'analyse de contenu thématique quantitative. En ce qui concerne la réalisation des entretiens semi-directifs, nous avons effectué une série de 8 entretiens avec des acteurs de la lutte contre le tabagisme et les maladies non transmissibles en Côte d'Ivoire. Lesdits acteurs peuvent être regroupés en 2 catégories qui sont les institutions publiques d'une part et les organisations non gouvernementales (ONG) d'autre part. Le choix de nous entretenir avec lesdits acteurs a été motivé par leur expertise reconnue en termes de lutte contre le tabagisme et les maladies non transmissibles en Côte d'Ivoire. Ainsi, nous avons eu des entretiens avec des représentants de 4 des 10 ONG membres du ROCTA-CI dont le vice-président de ce réseau d'ONG. De plus, les deux principales institutions publiques actives dans le cadre de la lutte contre le tabagisme et les maladies non transmissibles en Côte d'Ivoire, le Programme National de Lutte contre le Tabagisme, l'Alcoolisme et les autres addictions (PNLTA) et le Programme National de Prévention des Maladies Non Transmissibles (PNPMNT), ont également participé à nos entretiens.

\footnotetext{
${ }^{41}$ L'Afrique du Sud avait déjà adopté de nombreuses mesures préconisées par l'OMS dans la convention cadre pour la lutte antitabac avant son adoption en 2003.
} 
Tableau 1 : Récapitulatif des caractéristiques des enquêtés soumis aux entretiens semidirectifs

\begin{tabular}{|c|c|c|}
\hline INSTITUTIONS & $\begin{array}{l}\text { DOMAINES DE } \\
\text { COMPETENCE }\end{array}$ & $\begin{array}{c}\text { INTERLOCUTEURS ET } \\
\text { FONCTIONS AU SEIN DES } \\
\text { INSTITUTIONS }\end{array}$ \\
\hline CLUCOD (ONG) & $\begin{array}{l}\text { Membre du ROCTA-CI ; } \\
\text { Lutte contre le tabagisme }\end{array}$ & $\begin{array}{l}\text { M. Fousseni Tall, Sécrétaire } \\
\text { Général }\end{array}$ \\
\hline $\begin{array}{c}\text { JEUNESSE SANS } \\
\text { TABAC (ONG) }\end{array}$ & $\begin{array}{l}\text { Membre du ROCTA-CI ; } \\
\text { Lutte contre le tabagisme }\end{array}$ & $\begin{array}{c}\text { Docteur Mick Aloukré, } \\
\text { Président }\end{array}$ \\
\hline $\begin{array}{l}\text { ECOLE SANS TABAC } \\
\text { (ONG) }\end{array}$ & $\begin{array}{l}\text { Membre du ROCTA-CI } \\
\text { Lutte contre le tabagisme }\end{array}$ & $\begin{array}{c}\text { Docteur Francis Boli, } \\
\text { Président. Vice-président du } \\
\text { ROCTA-CI }\end{array}$ \\
\hline MULTA (ONG) & $\begin{array}{l}\text { Membre du ROCTA-CI } \\
\text { Lutte contre le tabagisme }\end{array}$ & $\begin{array}{l}\text { M. Ibrahim Timité, Chargé de } \\
\text { communication }\end{array}$ \\
\hline SAMSU (ONG) & $\begin{array}{c}\text { Lutte contre les maladies non } \\
\text { transmissibles et le } \\
\text { tabagisme }\end{array}$ & $\begin{array}{l}\text { Président du conseil } \\
\text { d'administration }\end{array}$ \\
\hline LICH (ONG) & $\begin{array}{c}\text { Lutte contre les maladies non } \\
\text { transmissibles et le } \\
\text { tabagisme }\end{array}$ & $\begin{array}{l}\text { M. Loukou Koffi, Directeur } \\
\text { coordinateur }\end{array}$ \\
\hline $\begin{array}{l}\text { PNLTA (Institution } \\
\text { publique) }\end{array}$ & $\begin{array}{c}\text { Lutte contre les maladies non } \\
\text { transmissibles et le } \\
\text { tabagisme }\end{array}$ & $\begin{array}{l}\text { Docteur Michel Yessoh, } \\
\text { Responsable service } \\
\text { épidémiologie-recherche }\end{array}$ \\
\hline $\begin{array}{l}\text { PNPMNT (Institution } \\
\text { publique) }\end{array}$ & $\begin{array}{l}\text { Lutte contre les maladies non } \\
\text { transmissibles et le tabagisme }\end{array}$ & $\begin{array}{c}\text { Docteur N'Guessan, Chargé de } \\
\text { la sensibilisation sur les } \\
\text { maladies non transmissibles }\end{array}$ \\
\hline
\end{tabular}

Source : Nos enquêtes, 2017, 2018

La recherche documentaire a été effectuée dans les bibliothèques de l'OMS et de l'Ambassade des Etats-Unis d'Amérique à Abidjan ainsi que sur le réseau internet. Le PNLTA et le PNPMNT ont également mis à notre disposition certains ouvrages internes que nous avons pu exploiter dans le cadre de la présente étude. Nous nous sommes ainsi procuré plusieurs ouvrages traitant de la thématique du tabagisme et des maladies non transmissibles. Par ailleurs, la recherche documentaire a servi de base à notre troisième méthode d'enquête qui est l'analyse de contenu thématique quantitative. Dans le cadre de la réalisation de cette dernière méthode d'enquête, nous avons utilisé deux catégories d'ouvrages qui sont les ouvrages relatifs à la lutte contre le tabagisme au plan mondial édictés par l'OMS et ceux relatifs au cadre juridique et aux instruments de lutte contre le tabagisme en Côte d'Ivoire. La première catégorie d'ouvrages était constituée de la convention-cadre de l'OMS pour la lutte antitabac et de la convention cadre de l'OMS pour la lutte antitabac. Directives pour l'application (voir tabl. 2). La deuxième catégorie d'ouvrages était constituée des différents décrets, arrêtés, circulaires, ordonnances et rapports relatifs à la lutte contre le tabagisme en Côte d'Ivoire (voir tabl. 3). 
Tableau 2 : Récapitulatif des mesures de la convention-cadre de l'OMS pour la lutte antitabac utilisées dans le cadre de l'analyse de contenu

\begin{tabular}{|c|c|}
\hline $\begin{array}{c}\text { MESURES RELATIVES A LA REDUCTION DE LA DEMANDE DE } \\
\text { TABAC }\end{array}$ & $\begin{array}{c}\text { NUMERO DE } \\
\text { L'ARTICLE }\end{array}$ \\
\hline Mesures financières et fiscales visant à réduire la demande de tabac & Article 6 \\
\hline Mesures autres que financières visant à réduire la demande de tabac & Article 7 \\
\hline Protection contre l'exposition à la fumée du tabac & Article 8 \\
\hline Réglementation de la composition des produits du tabac & Article 9 \\
\hline Réglementation des informations sur les produits du tabac à communiquer & Article 10 \\
\hline Conditionnement et étiquetage des produits du tabac & Article 11 \\
\hline Education, communication, formation et sensibilisation du public & Article 12 \\
\hline Publicité en faveur du tabac, promotion et parrainage & Article 13 \\
\hline $\begin{array}{c}\text { Mesures visant à réduire la demande en rapport avec la dépendance à l'égard } \\
\text { du tabac et le sevrage tabagique }\end{array}$ & Article 14 \\
\hline MESURES RELATIVES A LA REDUCTION DE L'OFFRE DE TABAC & \\
\hline Commerce illicite des produits du tabac & Article 15 \\
\hline Vente aux mineurs et par les mineurs & Article 16 \\
\hline $\begin{array}{c}\text { Fourniture d'un appui à des activités de remplacement économiquement } \\
\text { viables }\end{array}$ & Article 17 \\
\hline ENVIRONNEMENT ET RESPONSABILITE & Article 18 \\
\hline Protection de l'environnement et de la santé des personnes & Article 19 \\
\hline Responsabilité & Article 20 \\
\hline COOPERATION ET ECHANGE D'INFORMATIONS & Article 21 \\
\hline Recherche, surveillance et échange d'informations & Article 22 \\
\hline Notification et échange d'informations & \\
\hline Coopération dans les domaines scientifique, technique et juridique et \\
fourniture de compétences connexes
\end{tabular}

Sources : Réalisé à partir d'OMS (2003)

Tableau 3 : Récapitulatif du cadre juridique et des instruments de lutte contre le tabagisme en Côte d'Ivoire

\begin{tabular}{|c|c|}
\hline $\begin{array}{c}\text { CADRE JURIDIQUE ET INSTRUMENTS DE LUTTE CONTRE LE } \\
\text { TABAGISME EN COTE D'IVOIRE }\end{array}$ & $\begin{array}{c}\text { DATE D'ADOP- } \\
\text { TION }\end{array}$ \\
\hline $\begin{array}{c}\text { Décret 65-74 (marquage des tabacs à fumer, des cigares, des cigarettes et des } \\
\text { allumettes) }\end{array}$ & 1965 \\
\hline Décret 79-477 (Interdiction de fumer dans certains locaux à usage collectif) & 1979 \\
\hline Décret 92-487 (Etiquetage et présentation des denrées alimentaires) & 1992 \\
\hline Arrêté 24 MS/MC (Avertissement sur les emballages des produits du tabac) & 1998 \\
\hline Circulaire 891 (Avertissement sur les emballages des produits du tabac) & 1998 \\
\hline Arrêté 47 MC/MS (Avertissement sur les emballages des produits du tabac) & 1998 \\
\hline Ordonnance 2000-740 (Aménagement fiscalité spécifique sur les tabacs) & 2000 \\
\hline Décret 2010-08 (Ratification et publication de la convention-cadre de l'OMS) & 2010 \\
\hline Décret 2012-980 (Interdiction de fumer dans les lieux publics) & 2012 \\
\hline $\begin{array}{c}\text { Rapport affaire Diop C. contre Président de la République (Interdiction de } \\
\text { fumer dans les lieux publics) }\end{array}$ & 2013 \\
\hline
\end{tabular}

Source: Réalisé à partir des travaux de l'Observatoire du Tabac en Afrique Francophone (OTAF, 2017)

Dans le cadre de l'analyse de contenu thématique quantitative, nous avons dégagé 4 catégories d'analyses au niveau de la convention cadre de l'OMS pour la lutte antitabac. Au niveau de la convention cadre de l'OMS pour la lutte antitabac. Directives pour l'application, nous en avons dégagé 
7. Le choix des catégories d'analyses a été guidé par l'organisation des chapitres des ouvrages soumis à notre étude. En effet, lesdits ouvrages étaient initialement segmentés en chapitres qui classaient les articles de la convention-cadre de l'OMS pour la lutte antitabac selon des thèmes qui en faisaient la synthèse.

Tableau 4 : Récapitulatif des catégories d'analyses relatives à la convention cadre de l'OMS pour la lutte antitabac

\begin{tabular}{|c|c|c|c|c|}
\hline $\begin{array}{c}\text { Catégories } \\
\text { d'analyses }\end{array}$ & 1 & 2 & 3 & 4 \\
\cline { 2 - 5 } & $\begin{array}{c}\text { Réduction de } \\
\text { la demande } \\
\text { (articles 6 à } \\
14)\end{array}$ & $\begin{array}{c}\text { Réduction de } \\
\text { l'offre } \\
\text { articles 15 à } \\
17)\end{array}$ & $\begin{array}{c}\text { Environnement et } \\
\text { responsabilité } \\
\text { (articles 18 à 19) }\end{array}$ & $\begin{array}{c}\text { Coopération et } \\
\text { échange } \\
\text { d'informations } \\
\text { (articles 20 à 22) }\end{array}$ \\
\hline
\end{tabular}

Source : Réalisé à partir d'OMS (2003)

Tableau 5 : Récapitulatif des catégories d'analyses relatives à la convention cadre de l'OMS pour la lutte antitabac. Directives pour l'application

\begin{tabular}{|c|c|c|c|c|c|c|c|}
\hline Catégo- & 1 & 2 & 3 & 4 & 5 & 6 & 7 \\
\cline { 2 - 7 } ries & Direc- & Directi- & Directi- & Directi- & Directi- & Directi- & Directi- \\
d'ana- & tives & ves pour & ves pour & ves pour & ves pour & ves pour & ves \\
lyses & pour & l'article 8 & les & l'article & l'article & l'article & pour \\
& $\begin{array}{c}\text { articles 9 } \\
\text { l'arti- } \\
\text { cle 5.3 }\end{array}$ & & 11 & 12 & 13 & $\begin{array}{c}\text { l'arti- } \\
\text { cle 14 }\end{array}$ \\
\hline
\end{tabular}

Source : Réalisé à partir d'OMS (2013)

Dans le contexte de notre étude, nous avions pour unité d'information, toutes les propositions exprimant un avis sur une des catégories d'analyses. L'unité d'enregistrement pour sa part se rapportait au passage complet ou à la phrase relative à l'une des catégories d'analyses. De manière pratique, il s'est agi pour chacune des unités d'enregistrement dégagée dans la première catégorie d'ouvrages, de rechercher un équivalent dans au moins un des documents de la deuxième catégorie d'ouvrages. En d'autres termes, il s'agissait pour nous de faire correspondre chaque recommandation de la première catégorie d'ouvrages à une mesure contenue dans au moins un des documents de la deuxième catégorie d'ouvrages. Dans ce sens, nous avons obtenu pour chacune des catégories d'analyses explicitée des recommandations totalement appliquées, des recommandations partiellement appliquées et enfin des recommandations totalement inappliquées. Une recommandation totalement appliquée correspond à une recommandation inscrite dans un des documents de la première catégorie d'ouvrages et qui se retrouve dans son entièreté dans au moins un des documents de la deuxième catégorie d'ouvrages. Une recommandation partiellement appliquée correspond à une recommandation contenue dans un des documents de la première catégorie d'ouvrages et qui se retrouve en partie dans au moins un des documents de la deuxième catégorie d'ouvrages. 
Enfin, une recommandation totalement inappliquée est une recommandation contenue dans au moins un des documents de la première catégorie d'ouvrages et qui est absente de tous les documents de la deuxième catégorie d'ouvrages.

Nous notons par ailleurs que la norme veut que le cadre juridique et les instruments de lutte contre le tabagisme dont disposent les différents Etats signataires de la convention-cadre de l'OMS pour la lutte antitabac soient en adéquation avec les dispositions de ladite convention (OMS, 2003). Dans ce sens, une grande distorsion entre les deux catégories d'ouvrages soumis à l'analyse de contenu thématique quantitative dans le cadre de notre étude traduirait une inadéquation entre le cadre juridique et les instruments de lutte contre le tabagisme dont disposent la Côte d'Ivoire et les dispositions de la convention-cadre de l'OMS pour la lutte antitabac.

Le traitement et l'analyse des différentes données récoltées nous ont conduits à structurer la présente réflexion en deux principaux points : le degré de convergence entre le cadre juridique et les instruments de lutte contre le tabagisme en Côte d'Ivoire et les dispositions de la conventioncadre de l'OMS d'une part et les représentations des acteurs de la lutte contre le tabagisme d'autre part.

\section{Résultats et analyses}

Recherche de convergence entre le cadre juridique et les instruments de lutte contre le tabagisme en côte d'ivoire et les dispositions de la convention-cadre de l'OMS pour la lutte antitabac

L'analyse de la première catégorie de documents nous a permis de faire ressortir 100 recommandations adressées aux Etats signataires de la Convention-cadre de l'OMS pour la lutte antitabac et 376 recommandations adressées à ces mêmes Etats au niveau du rapport Convention-cadre de l'OMS pour la lutte antitabac. Directives pour l'application.

\section{Convention-cadre de l'OMS pour la lutte antitabac et cadre juridique et instruments de lutte contre le tabagisme en Côte d'Ivoire}

En ce qui concerne la Convention cadre de l'OMS pour la lutte antitabac, notre enquête nous a permis de constater que 20 recommandations contenues dans ladite Convention cadre sont totalement appliquées en Côte d'Ivoire. 28 autres des recommandations de ce document sont partiellement appliquées dans le pays et enfin 52 recommandations contenues dans cette convention-cadre sont totalement inappliquées en Côte d'Ivoire.

Ainsi, nous constatons que $20 \%$ des recommandations contenues dans la Convention cadre de l'OMS pour la lutte antitabac sont totalement appliquées en Côte d'Ivoire. Le pourcentage de recommandations de cette convention qui restent partiellement appliquées dans le pays est de $28 \%$. 
Enfin, nous constatons que 52\% des recommandations contenues dans ladite convention sont totalement inappliquées en Côte d'Ivoire.

Figure 1 : Suivi de la convention cadre pour la lutte antitabac en Côte d'Ivoire

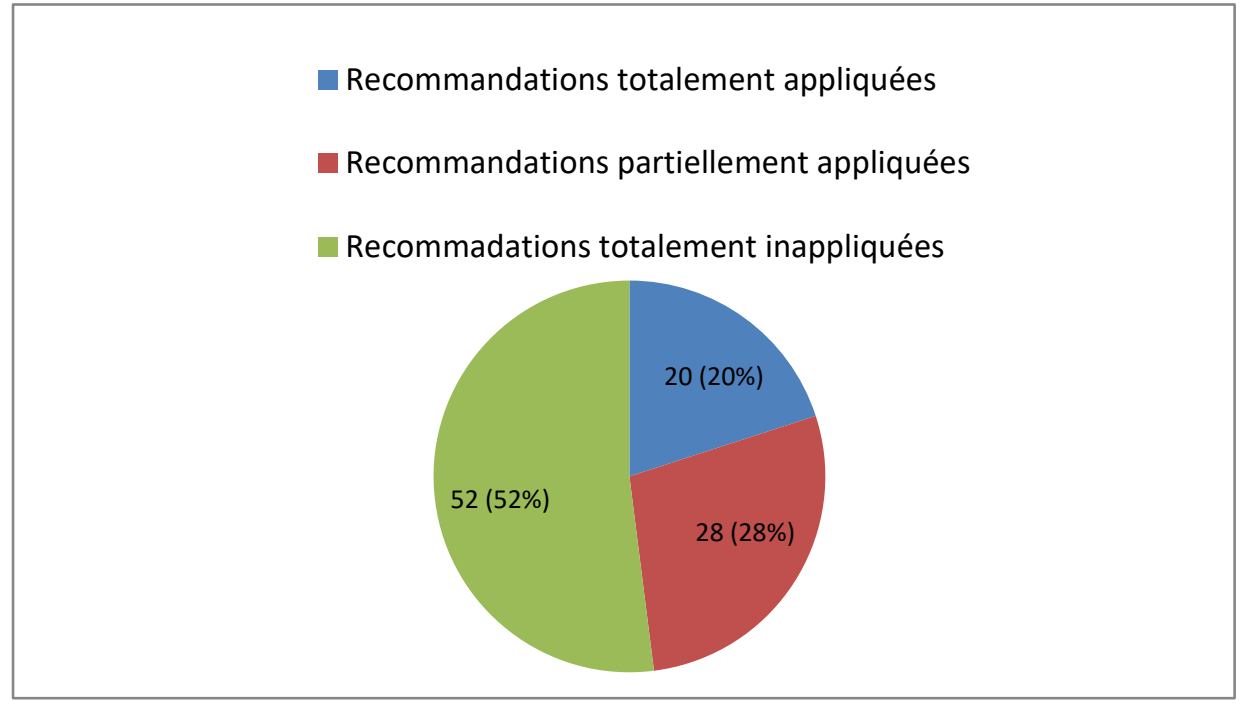

Source : Nos enquêtes, 2018

Convention-cadre de l'OMS pour la lutte antitabac. Directives pour l'application et cadre juridique et instruments de lutte contre le tabagisme en Côte d'Ivoire

$\mathrm{Au}$ niveau du rapport Convention-cadre de l'OMS pour la lutte antitabac. Directives pour l'application, nous avons pu mettre en évidence le fait que 62 des recommandations issues de ce rapport sont totalement appliquées en Côte d'Ivoire. Il existe également 62 autres recommandations dudit rapport qui sont partiellement appliquées en Côte d'Ivoire et enfin 252 des recommandations de ce rapport de l'OMS sont totalement inappliquées dans le pays.

Nous constatons que $17 \%$ des recommandations du rapport Convention-cadre de l'OMS pour la lutte antitabac. Directives pour l'application sont totalement appliquées en Côte d'Ivoire. $16 \%$ des recommandations que contient ledit rapport sont partiellement appliquées dans le pays. Enfin, le pourcentage de recommandations totalement inappliquées du rapport est de $67 \%$. 
Figure 2 : Convention cadre de l'OMS. Directives pour l'application en Côte d'Ivoire

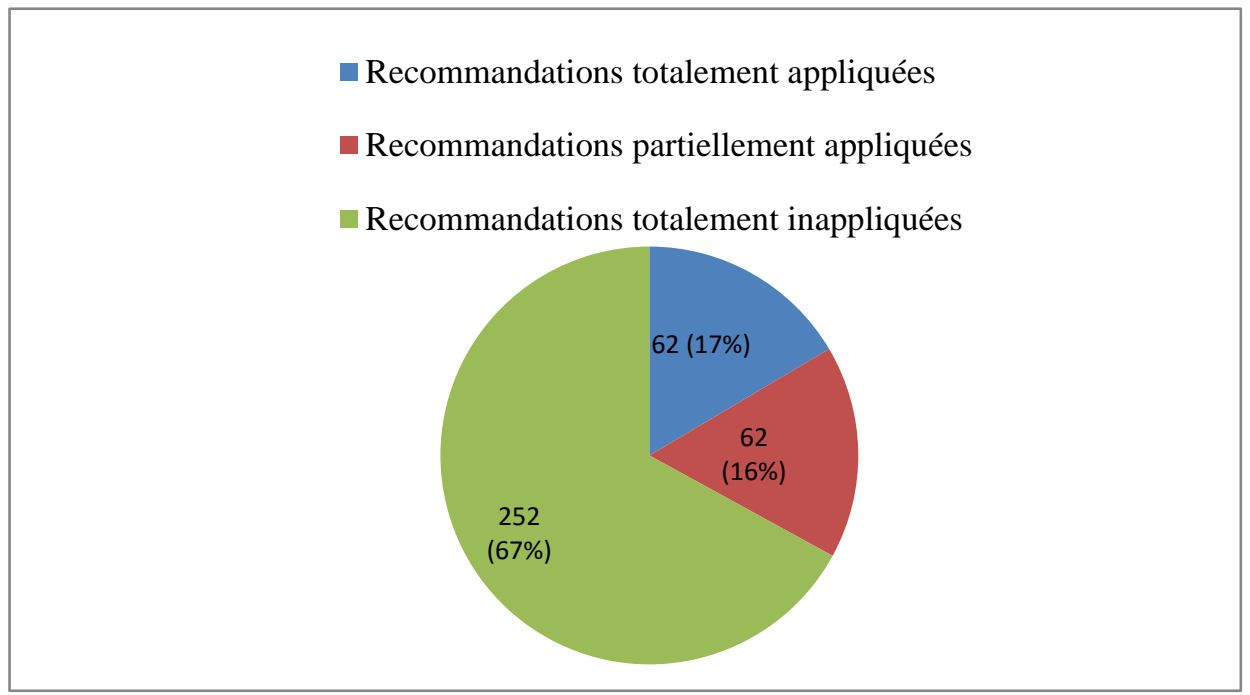

Source : Nos enquêtes, 2018

\section{Représentations des acteurs de la lutte contre le tabagisme en Côte d'ivoire}

Au niveau de l'adéquation entre le cadre juridique et les instruments de lutte contre le tabagisme de la Côte d'Ivoire et les recommandations de l'OMS en la matière

Les propos des enquêtés traduisaient globalement une insatisfaction en ce qui concerne le niveau d'application de la convention-cadre de l'OMS pour la lutte antitabac en Côte d'Ivoire. Ainsi, les enquêtés ont mis en avant dans leurs propos le fait que la Côte d'Ivoire ne disposait pas encore d'une loi antitabac en dépit de la réalisation de plaidoyer pour l'adoption d'une telle loi. Les propos suivants tenus par certains des enquêtés traduisent ce point de vue :

"Il n'existe pas de loi antitabac conforme aux dispositions de la convention cadre en Côte d'Ivoire [...], c'est cela le vrai problème de la lutte antitabac en Côte d'Ivoire [...], il existe juste des décrets ou arrêtés éparses et dépassés alors que la convention cadre que la Côte d'Ivoire a bien ratifié en 2010 recommande l'adoption d'une loi antitabac avec des dispositions pertinentes [...]. Jusqu'à présent, il n'y a pas de véritable architecture législative pour la lutte antitabac. A partir de là que pouvonsnous faire sur le terrain? Sur quelle base?"

"L'industrie du tabac fait tout pour entraver nos actions de sensibilisation contre le tabac [...] c'est ce genre de chose que la promulgation d'une loi antitabac doit régler dans le pays. Cette loi doit être adoptée et nous faisons pression sur le gouvernement pour que cette loi soit adoptée un jour. » 
"Il y a une grosse défaillance au niveau de la loi en Côte d'Ivoire [...], il faut une loi allant dans le sens de l'OMS [Loi antitabac conforme aux dispositions de l'OMS] en Côte d'Ivoire pour nous permettre d'améliorer notre action $[\ldots]$ »

"On [L'Etat de Côte d'Ivoire] a ratifié le traité [Convention cadre de l'OMS pour la lutte antitabac] et on ne l'applique pas. C'est un traité qui permet aux Etats d'aller plus loin que les recommandations de l'OMS, c'est bien signifié dans la convention cadre. Mais nous ne faisons même pas le minimum en votant la loi antitabac [...]»

\section{Au niveau des moyens financiers consacrés à la lutte contre le tabagisme}

Les personnes enquêtées ont identifié dans leurs propos la faiblesse des moyens financiers consacrés à la lutte antitabac en Côte d'Ivoire comme un facteur qui entraverait l'efficacité de la lutte contre le tabagisme en Côte d'Ivoire. Les propos des enquêtés suivants mettent en lumière cette situation :

"Les ressources [Ressources financières] dont nous disposons sont nettement inférieures à celles que consacre l'industrie du tabac au marketing de ses produits [...] dans ces conditions, nous n'évoluons pas sur un pied d'égalité $[\ldots] »$

«Nous faisons ce que nous pouvons parce que l'argent fait clairement défaut dans le cadre de la lutte contre le tabac [...] que ce soit au niveau des ONG comme la nôtre ou même du PNLTA, il y a de besoins énormes et pour l'instant, ces besoins ne sont pas comblés »

"Il serait bien que l'Etat accorde des ressources supplémentaires à la lutte contre le tabagisme [...] Il existe actuellement des subventions ministérielles accordées à certaines ONG mais elles sont trop faibles pour que nous puissions être vraiment efficaces sur le terrain»

"Les actions de sensibilisation que nous menons nécessitent des moyens financiers conséquents. Si on veut empêcher que la population ivoirienne ne soit confrontée à l'avenir à une augmentation des cancers et autres maladies cardiovasculaires entrainées par le tabagisme, il faut que les moyens consacrés par l'Etat à la lutte contre le tabac augmentent. Une chose est sûre, l'Etat sera emmené à l'avenir à consacrer de l'argent à la lutte contre le tabagisme [...], il faut que l'Etat choisisse entre consacrer plus d'argent à la prévention qui coûte un peu cher ou en consacrer à la lutte contre les conséquences du tabagisme que sont les cancers et là, c'est extrêmement cher $[\ldots] »$ 


\section{Discussion}

\section{Les limites de la lutte contre le tabagisme en Côte d'Ivoire}

Les résultats de notre enquête font ressortir l'existence d'un gap important entre les recommandations de l'OMS en matière de lutte antitabac et les dispositions contenues dans le cadre juridique et instruments de lutte contre le tabagisme en Côte d'Ivoire. Nous pouvons dans ce sens mettre en lumière le fait que seul un faible pourcentage des recommandations effectuées par l'OMS est totalement appliqué en Côte d'Ivoire. Ainsi, seulement $20 \%$ des dispositions de la convention cadre de l'OMS pour la lutte antitabac sont totalement appliquées en Côte d'Ivoire. Ce taux descend d'ailleurs à 17\% lorsqu'on considère la convention cadre de l'OMS pour la lutte antitabac. Directives pour l'application. Cette situation traduit donc le fait que le cadre juridique et instruments de lutte contre le tabagisme en Côte d'Ivoire présentent des lacunes importantes qui sont susceptibles d'expliquer le mal éprouvé par la Côte d'Ivoire à lutter contre le tabagisme.

En outre, les propos des acteurs de la lutte contre le tabagisme que nous avons rencontrés confirment le fait que le cadre juridique et les instruments de lutte contre le tabagisme en Côte d'Ivoire soient inadaptés à la lutte antitabac telle que la conçoit l'OMS. Dans ce sens, il ressort de notre étude que les institutions actives dans le cadre de la lutte contre le tabagisme en Côte d'Ivoire réclament l'adoption d'une loi antitabac dans le pays. Le fait que l'adoption de la loi antitabac ait régulièrement été évoquée par les acteurs de la lutte contre le tabagisme que nous avons rencontré n'est pas anodin. En effet, l'adoption d'une telle loi permettrait à la Côte d'Ivoire de combler en grande partie les défaillances présentées par son cadre juridique et ses instruments de lutte contre le tabagisme puisque la loi antitabac recommandée par l'OMS couvre globalement l'ensemble des articles de la convention cadre de l'institution.

Il importe également de mettre en avant le fait que les acteurs rencontrés s'accordent unanimement sur le fait qu'ils disposent de moyens insuffisants pour effectuer leurs actions relatives à la lutte contre le tabagisme en Côte d'Ivoire. Il faut en outre considérer le fait que l'OMS accorde une place importante à l'allocation de ressources financières suffisantes aux acteurs de la lutte contre le tabagisme. En effet, l'institution recommande aux Etats engagés dans la lutte antitabac à travers la ratification de la convention cadre de l'OMS d'apporter aux acteurs de la lutte antitabac, " un appui financier en faveur des activités nationales visant à atteindre l'objectif de la convention »(OMS, 2003, p.24). Cette recommandation illustre donc l'importance pour les acteurs de la lutte contre le tabac en Côte d'Ivoire de disposer de moyens financiers suffisants pour pouvoir mener à bien leurs missions. Cependant, cette préconisation de l'OMS semble ne pas avoir d'effet en Côte d'Ivoire où les acteurs de la lutte contre le tabagisme du 
pays font état du manque de moyens auquel ils font face dans leurs activités. Ces différentes situations concourent donc à réduire l'impact de la lutte contre le tabagisme en Côte d'Ivoire et expliquent la faiblesse des résultats obtenus dans cette lutte depuis plusieurs années.

Les résultats auxquels nous avons abouti convergent avec ceux d'autres recherches antérieures. Ainsi, Mvondo et Faton (2011) ont mis en lumière le fait que le dispositif légal du Bénin était incomplet et qu'il ne permettait pas de soutenir efficacement les actions de la société civile dans le cadre de la lutte antitabac. Au Burkina Faso également, l'ONG Aconta (2013) a exposé le fait que le cadre légal existant dans le pays était insuffisant pour parvenir à mener une lutte efficace contre le tabagisme. De tels résultats ont également été mis en lumière par Ben Amar et coll. (2017) qui ont montré les faiblesses du cadre législatif de la lutte antitabac en Tunisie et les problèmes qui en découlent dans la mise en œuvre des actions de lutte contre le tabagisme dans ce pays.

Par ailleurs, les travaux en France du Ministère du travail, de l'emploi et de la santé (2012) et ceux du Centre Sud (2014) montrent que l'insuffisance des financements accordés aux acteurs de la lutte antitabac est une situation courante dans les pays en développement.

\section{Recommandations}

\section{Au niveau des acteurs de la lutte contre le tabagisme}

Les acteurs de la lutte contre le tabagisme devraient continuer à mener des plaidoyers en faveur de l'adoption d'une loi antitabac comportant l'ensemble des dispositions pertinentes de la convention cadre de l'OMS pour la lutte antitabac. Par ailleurs, les plaidoyers déjà effectués dans le cadre d'une hausse des fonds accordés aux acteurs de la lutte contre le tabagisme devraient être également poursuivis auprès de l'Etat de Côte d'Ivoire, des institutions internationales et des grandes ONG transnationales engagées dans la lutte contre les maladies non transmissibles ou d'autres formes de maladies transmissibles pouvant être causées par le tabagisme telles que la tuberculose.

\section{Au niveau de l'Etat de Côte d'Ivoire}

L'Etat de Côte d'Ivoire devrait faciliter l'adoption d'une loi antitabac en Côte d'Ivoire. Par ailleurs, il serait opportun d'accroitre les ressources financières accordées aux acteurs de la lutte antitabac en Côte d'Ivoire. Cette action devrait être réalisée d'une part à travers une augmentation forte des taxes sur le tabac et d'autre part par la sollicitation de ressources financières extérieures auprès d'autres Etats signataires de la convention cadre de l'OMS pour la lutte antitabac. Ces différents leviers de mobilisation de ressources 
financières sont inclus dans la convention cadre de l'OMS pour la lutte antitabac (OMS, 2003).

\section{Perspectives d'amélioration de la lutte contre le tabagisme en Côte d'Ivoire}

Sur le plan fiscal, certaines actions ont commencé à être entreprises par l'Etat de Côte d'Ivoire à travers une hausse des taxes sur le tabac à partir de l'année 2016 (West Africa Rating Agency [WARA], 2016). Si cette action n'a pas permis d'atteindre les standards fiscaux recommandés par l'OMS (2018), elle pourrait néanmoins constituer un début de solution aux problèmes rencontrés dans le cadre de la lutte contre le tabagisme en Côte $\mathrm{d}^{\prime}$ Ivoire $^{42}$. Il faut également noter que 1'Union Economique et Monétaire Ouest Africaines (UEMOA, 2017) à laquelle appartient la Côte d'Ivoire a adopté la Directive n 01/2017/CM/UEMOA du 22 décembre 2017 portant harmonisation des législations des Etats Membres en matière de droits d'accises applicables aux tabacs. Les dispositions de ladite directive tendent à rehausser la fiscalité des produits du tabac dans la zone UEMOA en tenant réellement compte des conséquences sanitaires du tabagisme (Agence de Presse Africaine[APA], 2018). Cette action est donc conforme aux recommandations de l'OMS (APA, 2018). Ainsi, la Côte d'Ivoire sera probablement emmenée à rehausser à nouveau la fiscalité sur les produits du tabac avant la fin de l'année 2019 conformément à la nouvelle directive de l'UEMOA. Dans ce sens, la réalisation d'études sur l'impact de la hausse de la fiscalité sur le tabac en Côte d'Ivoire s'avèrera nécessaire à l'avenir pour appréhender l'évolution de la lutte contre le tabagisme dans le pays.

\section{Conclusion}

Au terme de la présente étude, il ressort que la lutte engagée contre le tabagisme en Côte d'Ivoire n'a pas encore atteint ses objectifs dans ce pays. Cette situation est causée par la persistance d'insuffisances au niveau du cadre juridique et des instruments de lutte contre le tabagisme dans le pays d'une part et par la faiblesse des ressources financières consacrées à la lutte contre le tabagisme d'autre part. Afin de remédier à cette situation, il serait opportun que l'Etat de Côte d'Ivoire prenne certaines mesures d'ordre fiscal et législatif.

${ }^{42}$ La réforme fiscale de 2015 est entrée en vigueur au début de l'année 2016. Elle a porté la fiscalité sur les produits du tabac au taux de $42 \%$ du prix de revient sortie usine alors que l'OMS recommande que les taxes sur les produits du tabac atteignent au moins $70 \% \mathrm{du}$ prix de vente final au consommateur. Ainsi, malgré la hausse fiscale, la base d'imposition et le taux appliqués ne permettent pas à la Côte d'Ivoire de respecter les recommandations de l'OMS. 


\section{Références:}

\section{Monographies}

1. Centre sud. (2014). Mécanismes de financement innovants : sources potentielles de financement de la convention cadre de l'OMS pour la lutte antitabac. (Document de recherche 54). Genève, Suisse : Auteur.

2. CRES. (2013). Projet régional de recherche action sur la taxation des produits du tabac en Afrique de l'ouest. Etat des lieux. Rapport de la Côte d'Ivoire. Abidjan, Côte d'Ivoire : CRES-CEDEAO.

3. Ministère du travail, de l'emploi et de la santé. (2012). Propositions pour une nouvelle politique de lutte contre le tabac. Rapport au Ministre du Travail, de l'Emploi et de la Santé. Paris, France: La documentation française.

4. Must, E., et Efroymson, D. (2003). Guide de l'usage des médias dans la lutte antitabac (traduit par A. Issa, K. Abdou et I. Maïga Djibo). Niamey, Niger : SOS Tabagisme-Niger.

5. Mvondo, P. et Faton, A. (2011). Mise en application de la convention cadre de l'OMS pour la lutte antitabac au Bénin. Rapport alternatif de la société civile. Cotonou, Bénin : ONG IECT.

6. NASAC. (2014). Prévention d'une épidémie de tabagisme en Afrique : Un appel pour une action efficace visant à appuyer le développement sanitaire, social et économique. Rapport de la Commission sur les effets néfastes du tabac sur la santé, l'économie et le développement de l'Afrique. Nairobi, Kenya : Auteur.

7. OMS. (2003). Convention-cadre de l'OMS pour la lutte antitabac. Genève, Suisse : Auteur.

8. OMS. (2013). Convention cadre de l'OMS pour la lutte antitabac. Directives pour l'application. Genève, Suisse : Auteur.

9. OMS. (2016). Le tabagisme chez l'adolescent. Copenhague, Danemark : Bureau régional de l'OMS pour l'Europe.

10. OMS. (2018). Taxation des produits $d u$ tabac. Repéré à http://www.who.int/tobacco/economics/taxation/fr/index1.html, consulté le 09 mars 2018.

11. ONG Aconta. (2013). Rapport parallèle de mise en ouvre des articles 5.3 ; 6 et 13 de la convention cadre de l'OMS pour la lutte antitabac au Burkina-Faso. Ouagadougou, Burkina Faso : Auteur.

12. WARA. (2016). Analyse Sitab S.A. Repéré à https://www.cgfbourse.com/news/annee2016/octobre2016/rapportanalyse--final-premiere-notation-sitab-ci-oct-2016.pdf, consulté le 29 juin 2017. 


\section{Article de périodique}

13. Ben Amar, W., Chakroun, A., Zribi, M., Khemekhem, Z., Ben Jemaa, F., et Maatoug, S. (2017). Dispositif législatif de lutte anti-tabagique en Tunisie : entre insuffisances et défaut d'application. Journal de l'information médicale de Sfax, (3), 21-26.

\section{Texte officiel}

14. UEMOA. (2017, 22 décembre). Directive $n^{\circ} 01 / 2017 / C M / U E M O A$ du 22 décembre 2017 portant harmonisation des législations des Etats Membres en matière de droits d'accises applicables aux tabacs. Repéré à http://www.uemoa.int/fr/type/tarif-exterieur-commun, consulté le 06 mars 2018.

\section{Pages web}

15. APA. (2017). Baisse du budget 2018 de la Commission de l'UEMOA. Repéré à http://mobile.apanews.net/fr/news/baisse-dubudget-2018-de-la-commission-de luemoa, consulté le 04 février 2018.

16. Arvers, P. (2017). Ces pays qui ont dissuadé les gens de fumer. Repéré

https://www.ouestfrance.fr/leditiondusoir/data/980/reader/reader.html \#!preferred/1/package/980/pub/981/page/7, consulté le 04 mai 2018.

17. CRDI. (2011). La lutte antitabac en Afrique du Sud : une stratégie gagnante. Repéré à https://www.idrc.ca/fr/article/la-lutte-antitabacen-afrique-du-sud-une-strategie gagnante, consulté le 21 janvier 2017.

18. OMS. (2017). Venir à bout du tabac pour améliorer la santé, l'environnement et le développement national. Journée mondiale sans tabac 2017. Repéré à http://www.who.int/fr/news-room/detail/30-052017-world-no-tobacco-day-2017-beating-tobacco-for-healthprosperity-the-environment-and-national-development, consulté le 13 mars 2018.

19. OTAF

(2017).

Législation.

Repéré

à http://www.otaf.info/legislation?field_theme_tid=All\&field_type_leg islation tid=All\&field_pays tid=13\&=Confirmer, consulté le 06 mai 2017. 